\title{
PERFORMANCE STUDY OF VALVELESS TRAVELING WAVE PIEZOELECTRIC PUMP IN MICROCHANNELS
}

\author{
Ajin Ghosh K K, Pradeep Kumar P, Bijudas C R, Seena V \\ ${ }^{1}$ Indian Institute of Space Science and Technology, Valiamala P.O., Thiruvananthapuram - 695547 \\ Kerala, India.
}

\begin{abstract}
A three dimensional piezoelectric coupled fluid structure interaction simulation have been done using COMSOL multiphysics software 5.3a for the performance study of a valveless traveling wave piezoelectric micropump (VTWP). A complete multiphysics computational domain with applied voltage as an input to the electrode patch to initiate and sustain the fluid motion has been benchmarked using data from Afrasiab et al.[1]. The benchmark model of the pump has a length of $5.2 \mathrm{~mm}$. The fluid channel is having a width of $200 \mu \mathrm{m}$ and depth of $300 \mu \mathrm{m}$. The top surface is a silicon diaphragm having a thickness of $6 \mu \mathrm{m}$ with a PZT$5 \mathrm{H}$ thin film layer of thickness $2.5 \mu \mathrm{m}$ over it. The VTWP is actuated by nine similar square electrode patches arranged along the axial direction of the channel. The working fluid is water. The unipolar sinusoidal peak to peak voltage of $20 \mathrm{~V}$ with a phase shift $120^{\circ}$ produces a traveling wave in the channel flow direction. Arbitrary Lagrangian Eulerian Method (ALE) has been employed for the fluid-structure interface deformation. In order to improve the performance of such pumps, the current study looks at two alternative configurations, one having a nozzle/diffuser (N/D) configuration and the other with a corrugated pump chamber. With a non-dimensional performance parameter and a non-dimensional flow ratio, the pumps with straight, N/D and corrugated configurations are compared for similar non-dimensional back pressure conditions. The pump with corrugated channel configuration shows better performance for the same back pressure.
\end{abstract}

KEY WORDS: Microchannel flow; Valveless traveling wave micropump; Piezoelectric; Fluid structure interaction.

\section{INTRODUCTION}

The miniaturization of flow control devices in micro systems has increased the quest for innovative solutions in recent years. Micropump classification based on the actuation mechanisms for general applications is discussed in [7], and for specific application like electronic cooling and bio-medical field are covered in [16] and [20]. Typically pumping in microscale can be realized by different method like mechanical, electrokinetic and magneto-kinetic, phase change mechanism and other novel technologies like flexural plate wave and electro-wetting[7, 14, 16, 20]. The valveless traveling wave piezoelectric micropump (VTWP ) [1, 10, 15] is a handy and reliable choice for low flow rate applications. Such pumps lend themselves for easy integration and can be usually configured to be inline with the fluid flow path in microchannels. There are a wide variety of microchannel flow applications in biomedical engineering and chemical engineering, where VTWP could be a good choice[2, 13, 18].

In 1988, Lintel described micromachined piezoelectric micropump by using a $10 \mathrm{~mm}$ diameter piezoelectric disc. The study addresses three passive silicon diaphragm check valves to control the back flow [9]. Several works on VTWP reported in the literature have been used for many applications handling liquids and gases 
Table 1 Design Parameters

\begin{tabular}{lc}
\hline Variable & Value \\
\hline Width of channel,W[1] & $200 \mu \mathrm{m}$ \\
Depth of channel,h[1] & $300 \mu \mathrm{m}$ \\
Smaller width of converging/ & \\
diverging channel, $W_{s}$ & $130 \mu \mathrm{m}$ \\
Angle of convergence/Divergence, $\alpha$ & $8^{\circ}$ \\
Length of converging/diverging section & \\
in the upstream and downstream section, $L_{d}$ & $500 \mu \mathrm{m}$ \\
Amplitude of corrugated channel,A & $40 \mu \mathrm{m}$ \\
Length of pump section, L[1] & $5.2 \mathrm{~mm}$ \\
\hline
\end{tabular}

in microchannels. A theoretical and experimental study of fluid dynamics of VTWP have been conducted by Bradly and White in 1994[4]. The design of VTWP and its influence on various parameters like channel height, wave amplitude and back pressure on the velocity profile and flow rate are discussed by Nguyen and White in their numerical optimization study[13]. The flow visualization of a piezoelectric cantilever actuated traveling wave micropump has been studied for membrane optimization[17]. The traveling wave micropump with sinusoidal actuated electrodes for microchannel with a flow area of $0.15 \mathrm{~mm}^{2}$ is found to typically produce a flow rate of $1.5 \mu \mathrm{l} / \min$ [15]. Typically, analysis of VTWPs found in the literature employ the computational domain for fluid structure interaction with wall displacement as an input to the model. Afrasiab et al. [3] reports a fluid structure interaction (FSI) using finite element method (FEM) simulation of both two and three dimensional models of VTWP and has benchmarked the experimental flow rate reported by Ogawa et al.[15]. The numerical simulation uses residual-based variational multiscale formulation with the stabilization parameter to avoid small time step convergence problems in FSI simulation [2]. A novel variant of traveling wave pump which mimics the peristaltic motion of fluid in a Teflon tube of diameter $1 \mathrm{~mm}$ is recently reported in[11]. The study reports a net pump outflow rate of $8.34 \mu \mathrm{l} / \mathrm{min}$ for the 16-electrode patch model.

The present study involves a complete piezoelectric coupled FSI simulation of a three dimensional model of micropump using COMSOL Multiphysics software. Prior to a complete model simulation of a VTWP, a preliminary numerical study using COMSOL multiphysics software has been carried out to ascertain the predictability of piezoelectric motion and FSI separately. This has been done with a typical piezoelectric bimorph deflection reported in [19] and a standard FSI problem of microcantilever motion in a fluid stream reported in [12] as the benchmark problems. Also, to ascertain the piezoelectric-FSI coupling, a piezoelectric bi-morph tip deflection in air and water reported in [8] has been benchmarked within 1\% numerical accuracy using COMSOL based fully coupled solver.

\section{NUMERICAL METHODOLOGY}

A typical VTWP is shown in Figure 1. The inlet is not restricted for back flow and the traveling wave is induced on the top wall through appropriate actuation of piezoelectric patches with a phase shift to produce a net outflow. Nine electrode patches are present on the top piezoelectric domain of the pump with three identical patches operating in a similar configuration. The numerical analysis of this straight configuration of VTWP is first established. Subsequently, two other modified configurations with the same hydraulic diameter, depth and length of pump chamber operating under similar back pressure conditions are carried out. Figure 2 shows the geometry of the proposed configurations. For the current study, the working fluid of the pump is taken as water. The pump is primarily made from silicon with piezoelectric material (PZT-5H) domain deposited to the top wall as a thin film. 


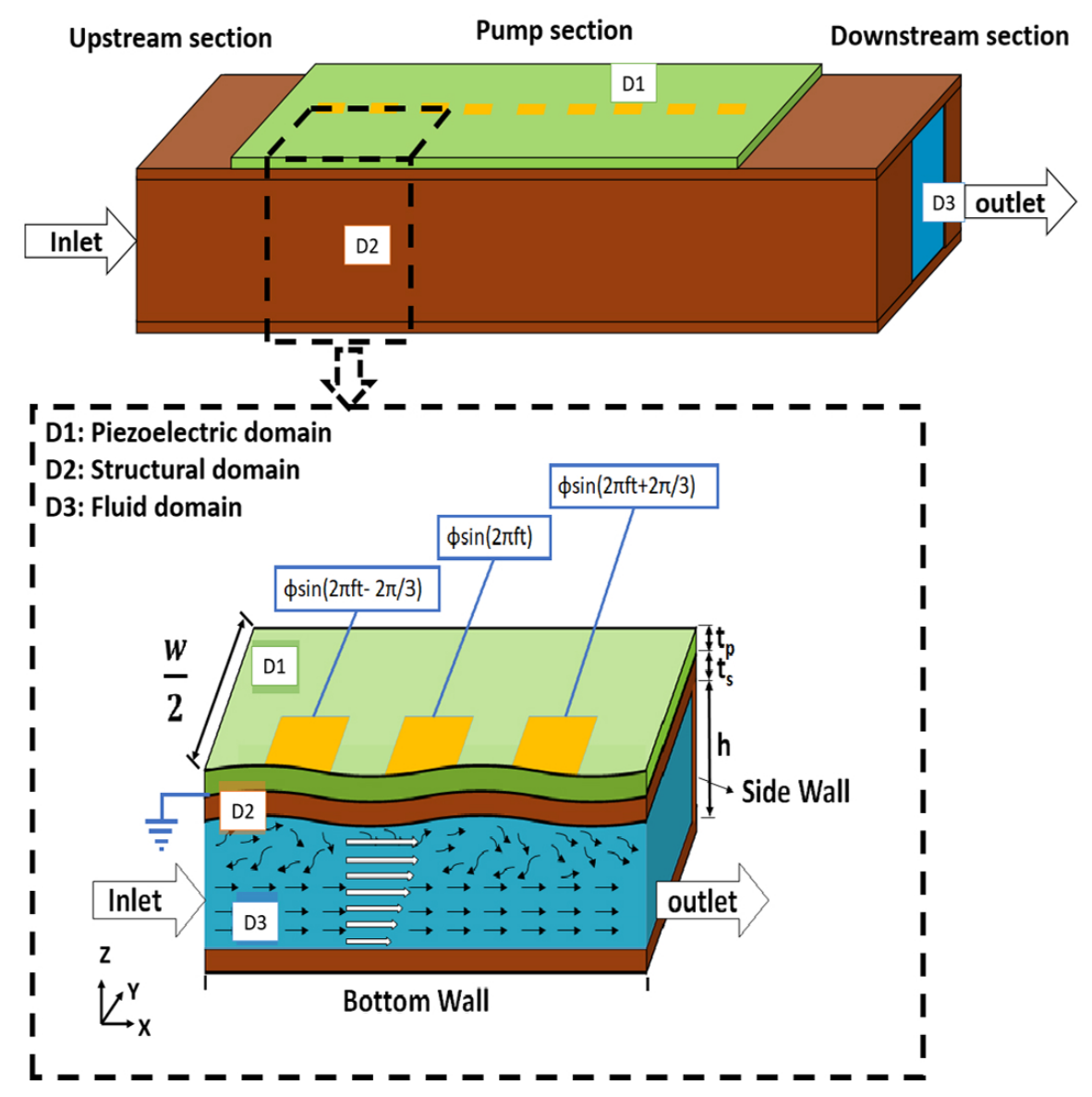

Fig. 1 A schematic of VTWP and the cross sectional view of the micropump section with three-electrode

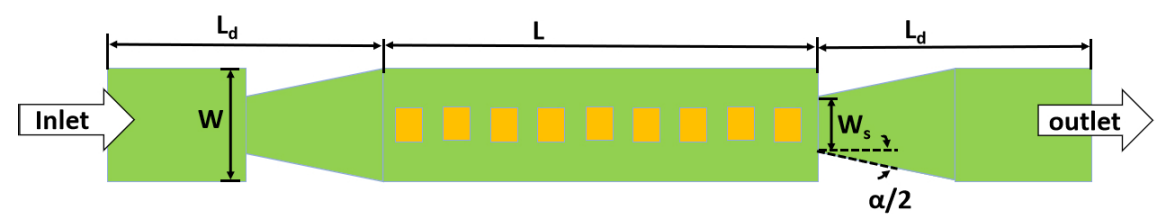

a)

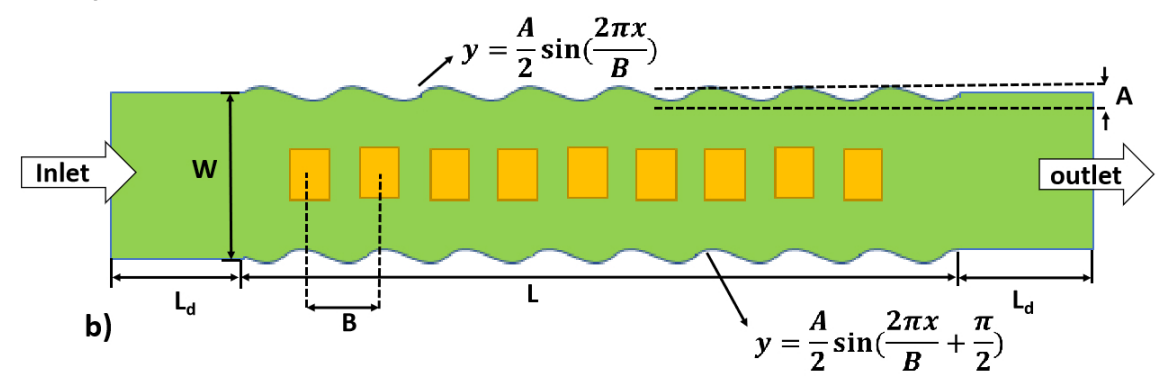

Fig. 2 Top view of the proposed design of micropump with nine electrodes. a) N/D configuration b)Corrugated configuration 
Table 2 Material properties of PZT-5H

\begin{tabular}{ll}
\hline Property & Value \\
\hline $\begin{array}{l}\text { Density } \\
\left(\mathrm{kg} / \mathrm{m}^{3}\right)\end{array}$ & 7800 \\
\hline $\begin{array}{l}\text { Piezoelectric coefficient } \\
\left(\mathrm{C} / \mathrm{m}^{2}\right)\end{array}$ & $\begin{array}{l}e_{31}=-6.5 \\
e_{33}=5.3 \\
e_{15}=17\end{array}$ \\
\hline & $c_{11}=139$ \\
Elasticity matrix & $c_{12}=80.2$ \\
& $c_{13}=84.6$ \\
$(\mathrm{GPa})$ & $c_{33}=115$ \\
& $c_{44}=22.98$ \\
& $c_{66}=23.47$ \\
\hline Relative permittivity & $\epsilon_{11}=1700$ \\
& $\epsilon_{33}=1470$ \\
\hline
\end{tabular}

\subsection{Computational domain.}

The configuration discussed in this section refers to the computational domain and numerical simulations of the straight configuration VTWP. The simulated pump output flow rate has been benchmarked. A similar methodology has been adopted for the two proposed configurations also. The straight configuration involves the 3D thin film piezoelectric domain, silicon pump wall and the fluid flow in the pump channel. The total length $(L)$ of the fluid channel is $5200 \mu \mathrm{m}$. The thickness of silicon $\left(t_{s}\right)$ is taken as $6 \mu \mathrm{m}$ and the thickness of PZT-5H $\left(t_{p}\right)$ is taken as $2.5 \mu \mathrm{m}$. The nine square electrode patches having a side length of 120 $\mu \mathrm{m}$, are located along the length of the channel with a $400 \mu \mathrm{m}$ center to center distance between adjacent electrodes[1, 15]. A dummy flow channel length of $1 \mathrm{~mm}\left(L_{d}\right)$ is added to the upstream and downstream sections of the VTWP [1]. Peak to peak voltage of $20 \mathrm{~V}$ is applied across the piezoelectric domain. The simulated net outflow characterizes the pump output. The piezoelectric and structural domain are appropriately meshed using hexahedral elements. The order of discretization is such that electrostatic domain has quadratic elements, the structural domain has quadratic Lagrangian elements and fluid domain has P2+P1 (quadratic velocity and linear pressure ) elements. The domain discretization and elements are chosen based on the preliminary numerical studies for the prediction of individual domains mentioned in the introduction. Typically higher order elements improves the prediction accuracy but with an increased computational expense. The dimensional parameters corresponding to each model are given in Table 1. The basic straight pump dimensions are chosen from Ogawa et al.[15]. In typical straight VTWP, the instantaneous flow generated is found as a bidirectional flow. To reduce such effects, a typical diaphragm type micropump usually adopts nozzle diffuser configuration to restrict back flow to some extent. Afrasiab et al.[1] proposed a VTWP with two asymmetric trapezoid obstacles at inlet and outlet. In this work, to reduce the instantaneous bidirectional flow, an alternate configuration is proposed. A straight micropump is modified with N/D arrangement at the inlet and outlet portion after the pump length. This N/D design is done based on diodicity value criteria given for converging/diverging microchannel in [6]. For the other alternate configuration, a corrugated channel is considered such that the effective diaphragm area is increased and corresponding actuation displacement will be larger as compared to a straight configuration. The corrugations are such that the average hydraulic diameter along the pump length is preserved. In the current study, the inbuilt piezoelectric effect coupling module and fluid structure interaction coupling module available in the COMSOL Multiphysics software[5] has been used.

\subsection{Boundary conditions.}

The domain consisting of piezoelectric thin film and the structural walls of the pump is considered as a composite structure. The thickness of the nine electrode patches being much smaller than the micropump 


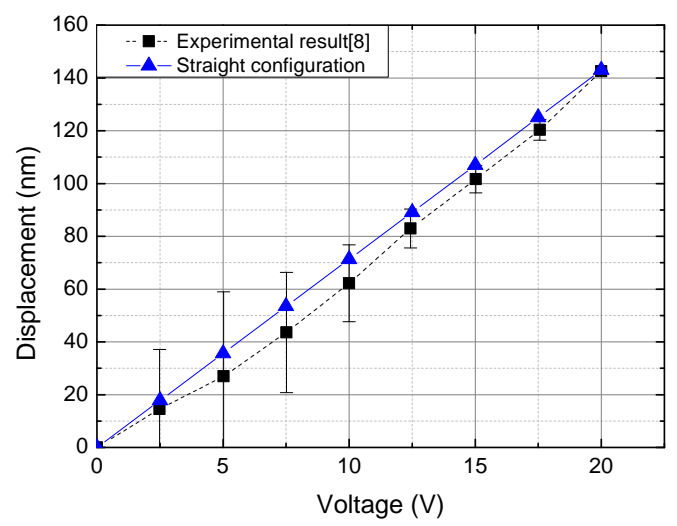

Fig. 3 Diaphragm displacement of the straight configuration validated with experimental results [15] for different voltage.

diaphragm wall thickness, the thickness of the electrode patch has been neglected for the current study. The electric potential is applied on each electrode and the interface between the silicon host structure domain and the piezoelectric material domain is grounded. The upper wall of the piezoelectric domain except the electrodes are electrically insulated. The side walls of diaphragm structure are considered to be fixed boundaries (ie; at $y=0, u_{s}=0$ and at $y=W, u_{s}=0$ ). And side wall of the diaphragm along the length is kept as a free boundary, where the stress is zero. The top wall of the fluid domain is in contact with the diaphragm which transfers the motion to the fluid. Hence the FSI interface condition is imposed on the diaphragm. On the inlet and outlet of the fluid domain is given an open boundary condition, ie; the net stress acting normal to the boundary surface is zero $\left(\left[-P I+\mu\left(\Delta V_{f}+\left(\Delta V_{f}\right)^{T}\right)\right] . \hat{n}=0\right)[5]$. For solving the fluid flow field, the open boundary condition describes boundaries that are open to large volumes of fluid which can both enter and leave the domain. The fluid domain walls are assigned to be in no-slip condition. The moving mesh displacements are solved from Laplace equation of moving mesh with implicit boundary conditions according to the fluid boundary in each direction. For subsequent performance study, three pump configurations are compared by numerical study with varying back pressure at the pump outlet as the boundary condition.

\subsection{Material properties}

Material properties of thin piezoelectric film and silicon have a detrimental effect on the predictability of piezoelectric induced traveling wave pump actuation. Typically, the nature of deposition of the thin film on silicon does not permit standard bulk property values of piezoelectric coefficients to be used. The characterization of actuator performance reported in [15] has been used to tune the piezoelectric coefficients. For the current study, it is seen that the piezoelectric coefficient $e_{33}$ has a value of $5.3 \mathrm{C} / \mathrm{m}^{2}$, which gives very close predictability of maximum displacement of the wall as against a typical bulk property value of $23.3 \mathrm{C} / \mathrm{m}^{2}$. The validation of maximum displacement for the different input voltage is shown in Figure 3. The piezoelectric material properties used for the current study are given in Table 2. The host structure is considered as isotropic silicon wafer with density $2300 \mathrm{~kg} / \mathrm{m}^{3}$, elastic modulus $170 \mathrm{GPa}$ and Poisson's ratio 0.215 .

\subsection{Benchmark}

The grid independence study has been conducted with quadrilateral mesh elements and a grid with 20400 elements in the fluid domain,1200 elements in an electrostatic domain and 4077 elements in host structural domain are found to give mesh independent solutions for the straight configuration. The simulations carried out in the Intel Xenon E5, 64 GB RAM system took 68 hours of computational time for 20 cycles of the input voltage. For the initial five cycles are ramping from zero initial voltage to maximum amplitude of input 


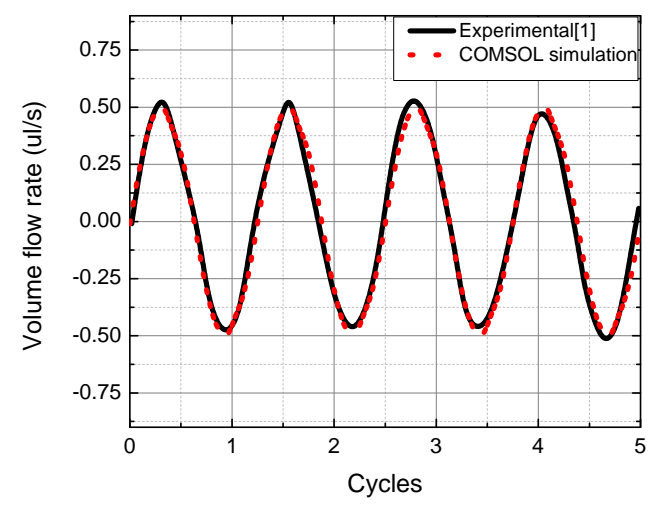

a)

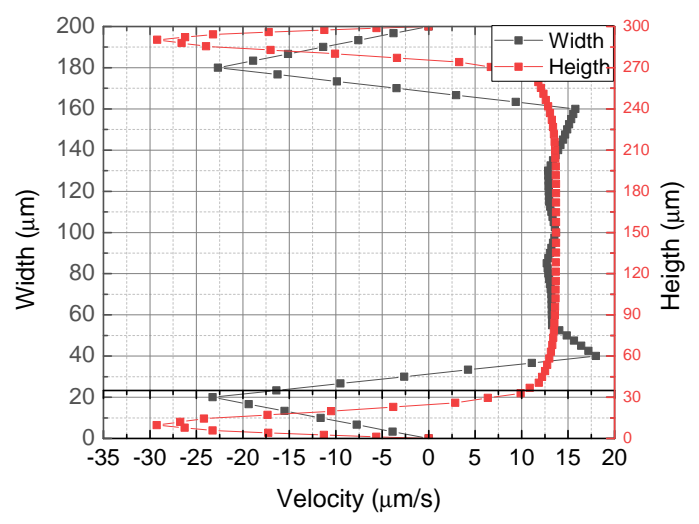

b)

Fig. 4 a) Comparison of outflow rate of current FEM simulation with experimental result [1]. b) Cyclic time averaged velocity profile at the center of the outlet of VTWP straight configuration.

sinusoidal voltage to avoid convergence problems. The initial state fluid and solid variables also set to zero and the transient behavior ramp-up with the actuation of diaphragm. After the first five cycles, a steady-state periodic solution is obtained. Typically, VTWPs work in high frequency and hence time step required to capture the motion will be very small. The fully coupled problem is solved by using the direct method with a time step size of 1/80 times of time period of the input voltage.

As the first step of the analysis, the numerical method is validated for the straight micropump reported in [1]. The outlet flow rate of current simulation is compared with the benchmark model as shown in Figure 4a). The axial time averaged net flow rate calculated over one cycle shows a deviation of less than $1 \%$ with experimental results. The Figure $4 \mathrm{~b}$ ) shows the cyclic time averaged velocity at the centerline of the outlet of the micropump in width and depth direction.

\subsection{Performance characterization}

In order to compare the pump performance of different configurations proposed, a non-dimensional performance parameter $E^{*}$ is defined. Also to compare the flow rates, a non-dimensional parameter, flow ratio $Q^{*}$ is defined. Both these parameters are compared for the three pump configurations working under similar non-dimensional back pressure $P^{*}$ conditions. The non-dimensional parameters are defined as,

$$
\begin{gathered}
E^{*}=\frac{\left(P_{b}-P_{r e f}\right)\left(Q_{n e t}\right)}{\phi_{r m s} I_{r m s}} \\
P^{*}=P_{b} / P_{r e f}
\end{gathered}
$$

The parameter $E^{*}$ depict the overall efficiency of the system. This is defined as the ratio of the electric input power supplied to the pump to output fluid power produced due to pumping action. The other parameters flow ratio $Q^{*}$, and non-dimensional back pressure $P^{*}$ are used to predict the flow characteristic of the pump. $Q^{*}$ is defined as the ratio of time averaged flow velocity at the outlet to the inlet.

$$
\begin{gathered}
Q^{*}=\frac{\overline{Q_{i}}}{\overline{Q_{o}}} \\
\overline{Q_{a}}=\frac{1}{\left(t_{2}-t_{1}\right)} \int_{t_{1}}^{t_{2}} Q_{a} d t
\end{gathered}
$$

Where $a=i$, for inlet and $a=o$, for outlet 


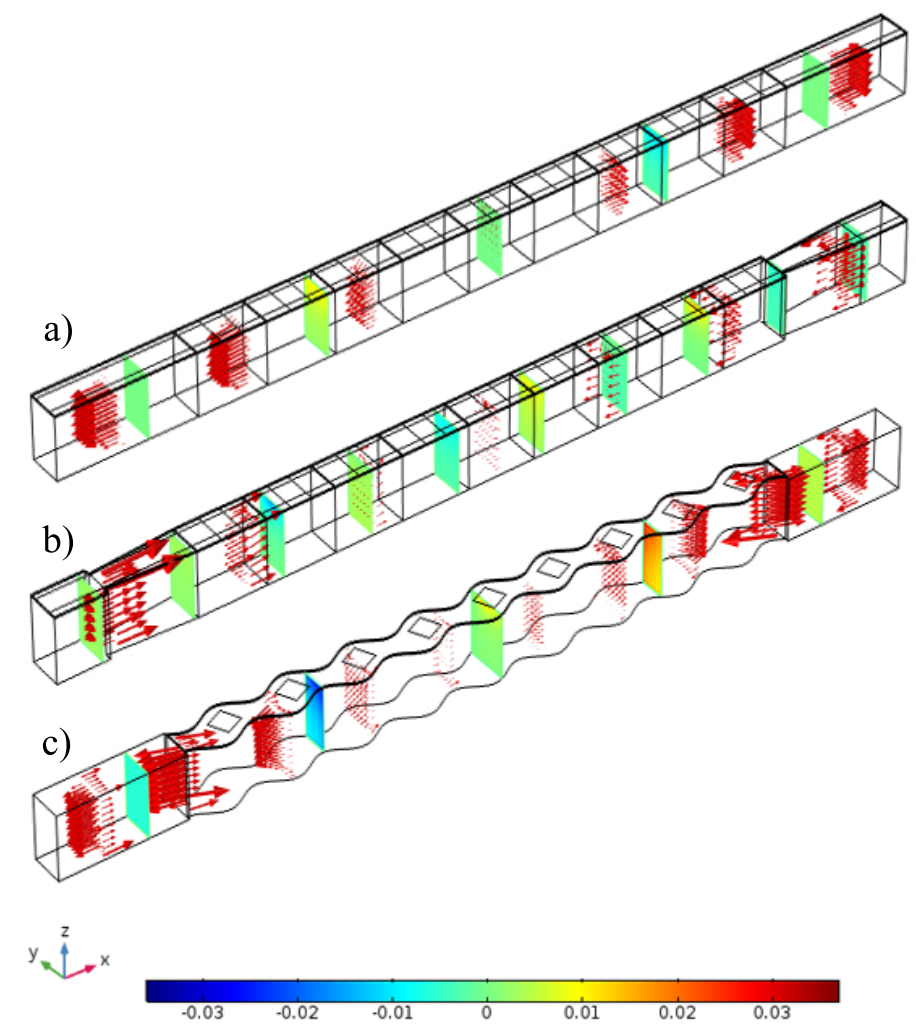

Fig. 5 Cyclic time averaged velocity vector and instantaneous velocity contour at the end of the $10^{\text {th }}$ cycle (in $\mathrm{m} / \mathrm{s}$ ). a)straight configuration b)N/D configuration c) Corrugated configuration

Where the $\overline{Q_{a}}$ is the time averaged inlet or outlet flow and $Q_{a}$ is the instantaneous flow.

\section{RESULTS AND DISCUSSION}

The pumping action created by the piezoelectric patches generates a traveling wave along the top wall causing a sinusoidal flow rate between the inlet and outlet. Figure 5 provides the time averaged velocity vector and instantaneous velocity contour at the end of the 10th cycle for all the three configurations. These instantaneous velocity vectors indicate instantaneous flow in both the directions. However, a net time averaged flow rate is obtained towards the outlet direction. The net cyclic flow rate of corrugated and N/D configurations are 2.19 and 3.9 times higher than the straight channel respectively. Figure 6, 7 and 8 indicate the non-dimensional pressure contour and velocity contour for straight, N/D and corrugated configurations. The pressure variations are seen to be analogous to the wall fluctuations. Figure 9 presents the plot of channel top wall displacements at the fifth patch location against $1000 \mathrm{~Pa}$ back pressure. The figure shows a displacement of the corrugated diaphragm is 1.23 times greater than the straight and N/D configuration. This is because of corrugated diaphragm area is 1.02 times greater than the area of straight or N/D configuration. Since the pump domain is hydraulically equivalent in all three cases, the corrugated pump diaphragm seen to have greater flexibility in the pumping process. The performance analysis conducted against the back pressure for different outlet pressure shows that the net outflow is reduced in proportion to the increased back pressure. The performance of the pump against the back pressure is quantified and plotted in terms of the non-dimensional parameter in the Figure 10. Figure 10a) shows the variation of effective energy conversion with respect to an increase in the back pressure. Figure 10b) shows that the net outflow for a cycle can be increased with both corrugated and N/D configuration as compared to straight channel configuration. For the considered back pressure conditions the corrugated pump configuration shows a better performance. At reasonably higher values of back pressure 


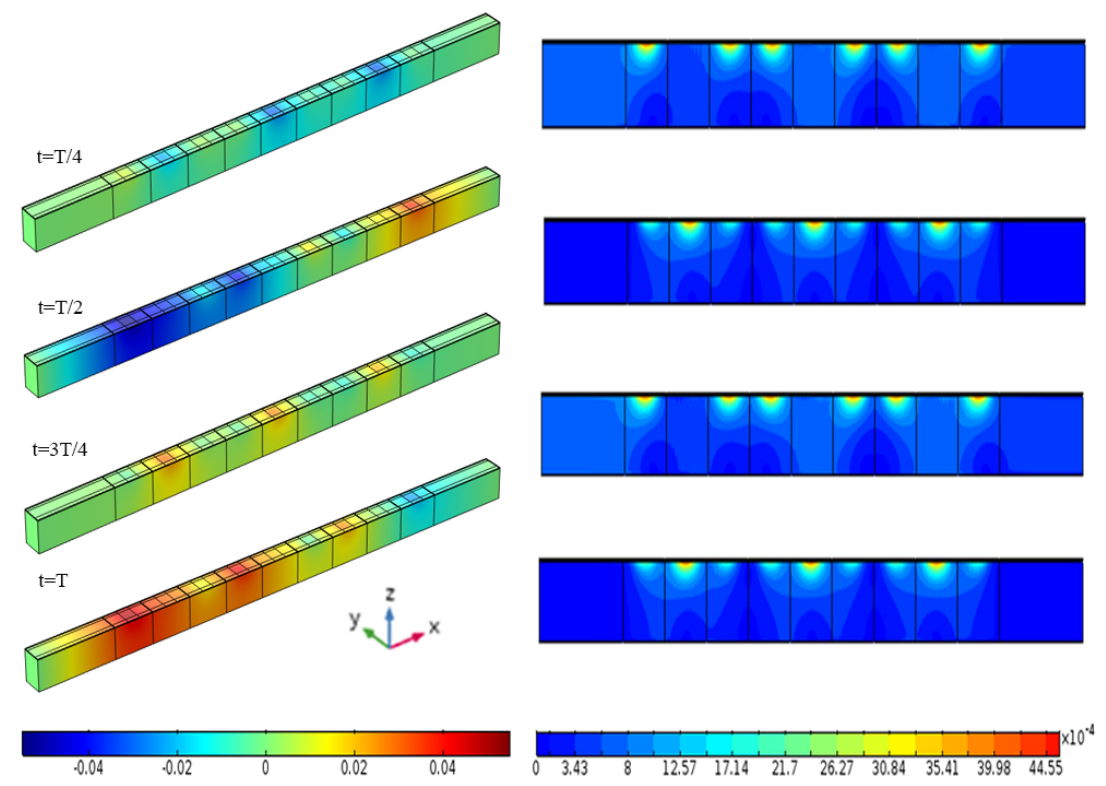

Fig. 6 The left side of the figure shows non-dimensional pressure contour $\left(P^{*}=\frac{P}{P_{r e f}}\right)$ and the right side of the figure shows non-dimensional velocity contour $\left(V^{*}=\sqrt{\frac{U}{\frac{P_{r e f}}{\rho}}}\right)$ at midsection along the flow direction in the straight configuration micropump at different instants of a cycle

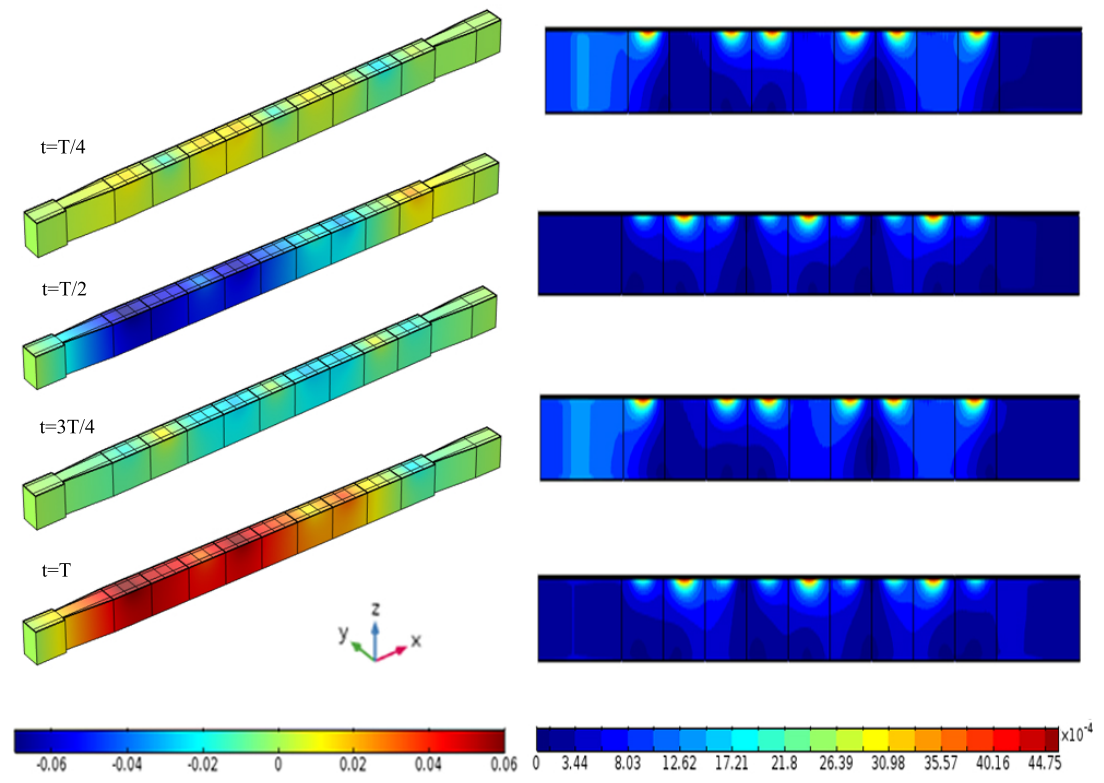

Fig. 7 The left side of the figure shows non-dimensional pressure contour $\left(P^{*}\right)$ and the right side of the figure shows non-dimensional velocity contour $\left(V^{*}\right)$ at midsection along the flow direction in the N/D configuration micropump at different instants of a cycle 


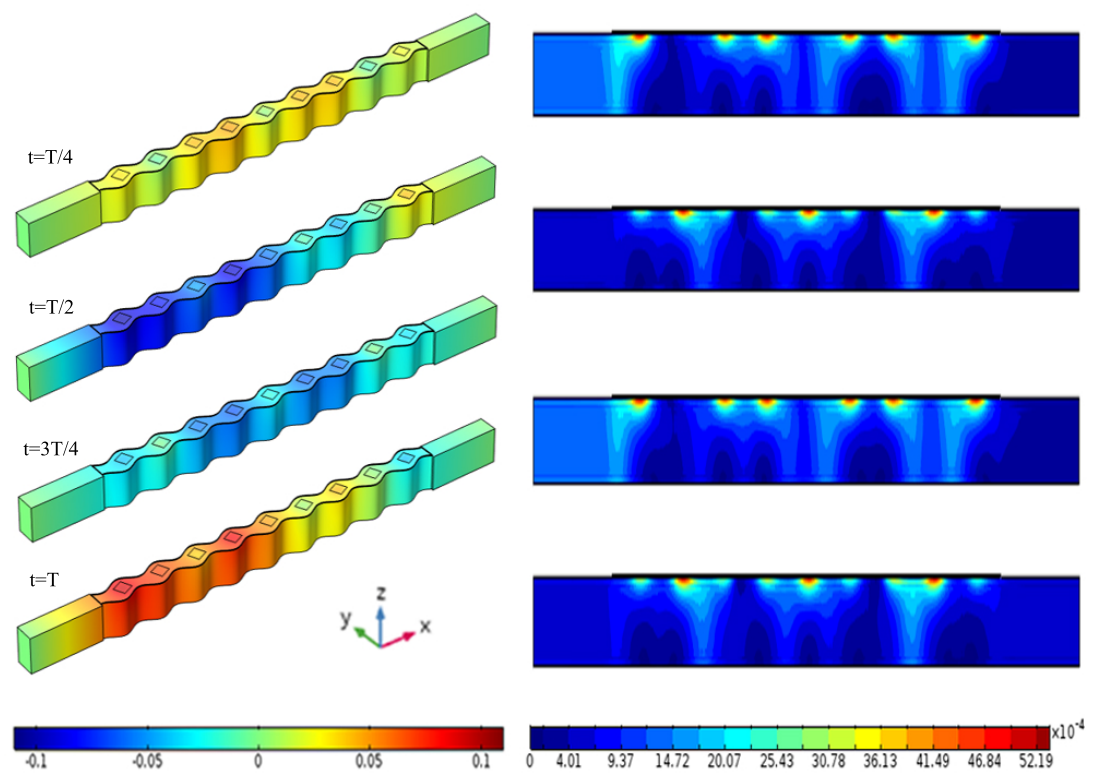

Fig. 8 The left side of the figure shows non-dimensional pressure contour $\left(P^{*}\right)$ and the right side of the figure shows non-dimensional velocity contour $\left(V^{*}\right)$ at midsection along the flow direction in the Corrugated configuration micropump at different instants of a cycle

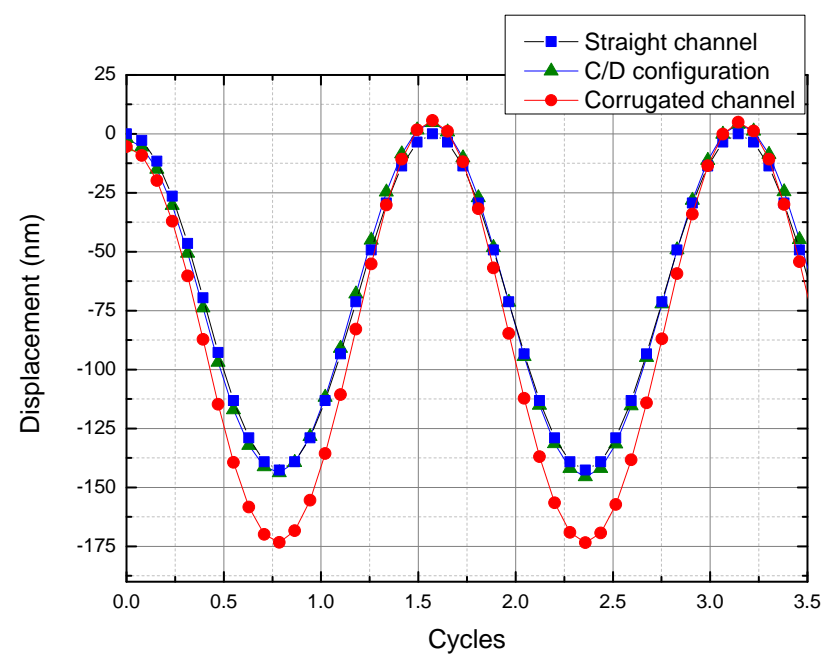

Fig. 9 Transverse displacement of three different configurations at the mid- electrode center at $1000 P a$ back pressure applied at the outlet boundary. 


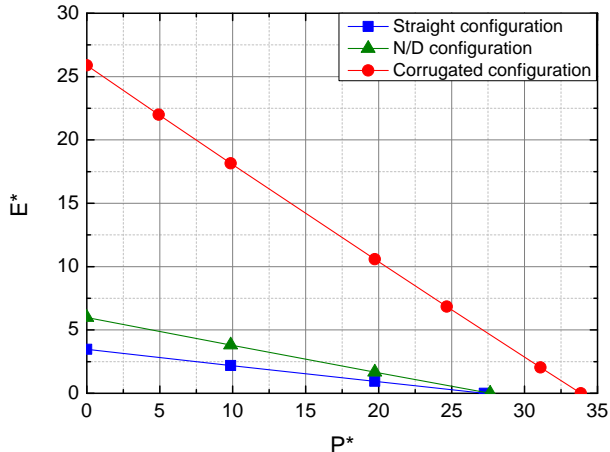

a)

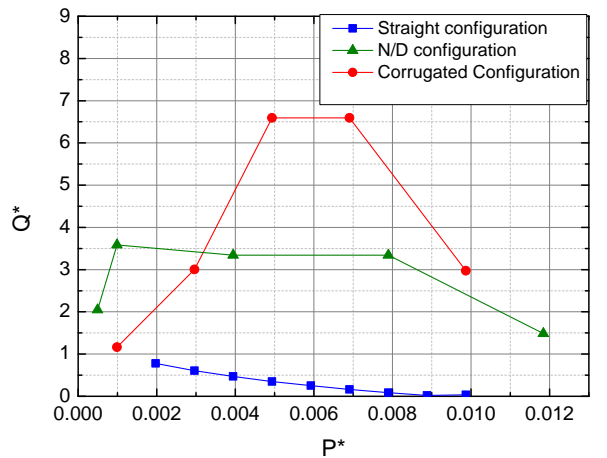

b)

Fig. 10 a)Performance parameter versus non-dimension back pressure plot b) Flow ratio versus nondimensional back pressure for different configuration at same input peak to peak voltage of $20 \mathrm{~V}$

one could size the corrugated configuration to give a higher flow rate as well.

\section{CONCLUSION}

Typically, the design analysis of MEMS thin film devices by numerical method involves multiphysics fields that are coupled. The present work assesses the performance of three configurations of VTWP. The main difficulty in design analysis are unknown material property and variations in the proposed design dimension from the actual one. For the current work, $e_{33}$ value in the piezoelectric coefficient matrix has been appropriately tuned with the published experimental results [15] of the piezoelectric diaphragm pump. With these as a basis, complete multiphysics simulations of VTWP have been validated using a strongly coupled FSI direct solver. The current study shows corrugated channel displacement to be $23 \%$ higher than the straight microchannel pump at $20 \mathrm{~V}$. This shows better flexibility for the pumping operation. The non-dimensional parameters $E^{*}$ (performance parameter), $Q^{*}$ (flow rate ratio) and $P^{*}$ (non-dimensional back pressure) have been used to assesses the performance of the three configurations. The current study indicates better performance and improved flow for corrugated configuration under the conditions of similar back pressure and hydraulically equivalent dimensions. In VTWP the performance strongly depends on the nature of actuation induced to the piezoelectric patch. Also, the limits of actuation in terms of amplitude and choice of frequency need to be ascertained through a detailed structural modal analysis. However, the present coupled analysis presented provides a typical piezoelectric characterization for the operation of VTWP.

\section{NOMENCLATURE}

$\begin{array}{llllll}\rho & \text { Density of the material } & \left(\mathrm{kg} / \mathrm{m}^{3}\right) & \phi & \text { Electric potential } & (V) \\ u & \text { Displacement of structure } & (\mathrm{m}) & E^{*} & \text { Non-dimensional Performance parameter } & (-) \\ \sigma & \text { Stress tensor } & \left(\mathrm{N} / \mathrm{m}^{2}\right) & P^{*} & \text { Non-dimensional back pressure } & (-) \\ C & \text { Elastic constants tensor } & \left(\mathrm{N} / \mathrm{m}^{2}\right) & Q_{n e t} & \text { Net time averaged flow rate } & \left(\mathrm{m}^{3} / \mathrm{s}\right) \\ S & \text { Strain tensor } & (\mathrm{m} / \mathrm{m}) & \phi_{r m s}^{*} & \text { Root mean square voltage } & (V) \\ D & \text { Displacement current } & \left(\mathrm{C} / \mathrm{m}^{2}\right) & I_{r m s}^{*} & \text { Root mean square velocity } & (A) \\ q & \text { Electric body charge } & (\mathrm{m}) & V & \text { Velocity } & (\mathrm{m} / \mathrm{s}) \\ E & \text { Electric field intensity } & (\mathrm{V} / \mathrm{m}) & v^{m} & \text { Mesh velocity } & (\mathrm{m} / \mathrm{s}) \\ e & \text { Piezoelectric stress constant } & \left(\mathrm{C} / \mathrm{m}^{2} \text { or } N / V m\right) & \mu & \text { Viscosity } & (\mathrm{m}) \\ \epsilon & \text { Electrical permittivity } & (F / m) & P & \text { Fluid pressure } & \left(\mathrm{N} / \mathrm{m}^{2}\right) \\ t & \text { Thickness } & (\mathrm{m}) & b & \text { Body force per unit mass } & \left(\mathrm{m} / \mathrm{s}^{2}\right)\end{array}$




\section{TFEC-2020-32042}

\section{REFERENCES}

[1] Afrasiab, H., Movahhedy, M., and Assempour, A., "Proposal of a new design for valveless micropumps," Scientia Iranica, 18(6), pp. 1261-1266, (2011).

[2] Afrasiab, H. and Movahhedy, M. R., "Treatment of the small time instability in the finite element analysis of fluid structure interaction problems," International Journal for Numerical Methods in Fluids, 71(6), pp. 756-771, (2013).

[3] Afrasiab, H., Movahhedy, M. R., and Assempour, A., "Fluid-structure interaction analysis in microfluidic devices: A dimensionless finite element approach," International Journal for Numerical Methods in Fluids, 68(9), pp. 1073-1086, (2012).

[4] Bradley, C. and White, R., "Acoustically driven flow in flexural plate wave devices: theory and experiment," 1994 Proceedings of IEEE Ultrasonics Symposium, Vol. 1, IEEE, pp. 593-597, (1994).

[5] COMSOL, (2017). COMSOL Multiphysics Reference Manual,version 5.3”, COMSOL, Inc,. www.comsol.com.

[6] Duryodhan, V. S., Singh, S. G., and Agrawal, A., "Liquid flow through converging microchannels and a comparison with diverging microchannels," Journal of Micromechanics and Microengineering, 24(12), pp. 125002, (2014).

[7] Iverson, B. D. and Garimella, S. V., "Recent advances in microscale pumping technologies: a review and evaluation," Microfluidics and nanofluidics, 5(2), pp. 145-174, (2008).

[8] Kaneko, S., Hong, G., Mitsume, N., Yamada, T., and Yoshimura, S., "Numerical study of active control by piezoelectric materials for fluid-structure interaction problems," Journal of Sound and Vibration, 435, pp. 23-35, (2018).

[9] Lintel, H. T. G. V., “A piezoelectric micropump based on micromachining silicon.,” Sensors and Actuators., 15(1), pp. 153-167, (1988).

[10] Liu, G. and Zhang, W., (2018). Springer, Traveling-Wave Micropumps. Springer, pp. 1017-1035.

[11] Na, Y.-m., Lee, H.-s., and Park, J.-k., "Fabrication and experiment of piezoelectric pump imitating peristalsis," Journal of Mechanical Science and Technology, 32(10), pp. 4737-4745, (2018).

[12] Namkoong, K., Choi, H., and Yoo, J., "Computation of dynamic fluid-structure interaction in two-dimensional laminar flows using combined formulation," Journal of Fluids and Structures, 20(1), pp. 51 - 69, (2005).

[13] Nguyen, N. and White, R., "Design and optimization of an ultrasonic flexural plate wave micropump using numerical simulation," Sensors and Actuators A: Physical, 77(3), pp. 229-236, (1999).

[14] Nguyen N, Huang X, C. T., "Mems-micropumps: A review.," ASME. J. Fluids Eng., 124(2).(1), pp. 384-392, (2002).

[15] Ogawa, J., Kanno, I., Kotera, H., Wasa, K., and Suzuki, T., "Development of liquid pumping devices using vibrating microchannel walls," Sensors and actuators A: Physical, 152(2), pp. 211-218, (2009).

[16] Singhal, V., Garimella, S. V., and Raman, A., "Microscale pumping technologies for microchannel cooling systems," Applied Mechanics Reviews, 57(3), pp. 191-221, (2004).

[17] Suzuki, T., Hata, H., Shintaku, H., Kanno, I., Kawano, S., and Kotera, H., "Visualization and optimization for fluid flow of traveling wave micropump using micropiv and numerical simulation," Proceedings of microTAS2005, pp. 1108-1110, (2005).

[18] Tabak, A. F. and Yesilyurt, S., "Simulation-based analysis of flow due to traveling-plane-wave deformations on elastic thin-film actuators in micropumps," Microfluidics and Nanofluidics, 4(6), pp. 489-500, (2008).

[19] Wang, S., "A finite element model for the static and dynamic analysis of a piezoelectric bimorph," International Journal of Solids and Structures, 41(15), pp. 4075-4096, (2004).

[20] Wang, Y.-N. and Fu, L.-M., "Micropumps and biomedical applications-a review," Microelectronic Engineering, 195, pp. 121138, (2018). 